\title{
Explaining $g-2$ anomalies in two Higgs doublet model with vector-like leptons
}

\author{
Eung Jin Chun and Tanmoy Mondal \\ Korea Institute for Advanced Study, \\ Seoul 02455, Korea \\ E-mail: ejchun@kias.re.kr, tanmoy@kias.re.kr
}

\begin{abstract}
We consider the two Higgs doublet model (2HDM) along with a generation of vector-like lepton doublet and singlet to explain the observed discrepancies in the electron and muon anomalous magnetic moments. The type-X (lepton-specific) $2 \mathrm{HDM}$ can allow a light pseudo-scalar which is known to explain the muon anomalous magnetic moment at two-loop. Such a light particle induces a sizable negative contribution to the electron anomalous magnetic moment at one-loop in the presence of vector-like leptons evading all the experimental constraints.
\end{abstract}

KEYwords: Phenomenological Models

ARXIV EPRINT: 2009.08314 


\section{Contents}

1 Introduction 1

2 The model 3

2.1 Couplings of the scalars and fermions 5

2.2 Couplings to gauge bosons 5

3 Electron and muon anomalous magnetic moment $\quad 6$

3.1 Electron $(g-2) \quad 6$

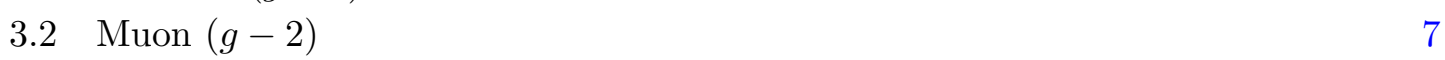

4 Constraints from precision observables $\quad 8$

4.1 Constraints from the $Z$ pole measurements 8

$\begin{array}{lll}4.2 & \text { Constraints from oblique corrections } & 9\end{array}$

5 Results and discussion $\quad 9$

$\begin{array}{lll}5.1 & \text { Results for electron }(g-2) & 10\end{array}$

$\begin{array}{ll}5.2 & \text { Results for muon }(g-2) \\ \end{array}$

6 Collider phenomenology 13

$\begin{array}{llr}7 & \text { Conclusion } & 14\end{array}$

\section{Introduction}

The precise determination of electron and muon anomalous magnetic moment, both theoretical and experimental, is an important test for the Standard Model (SM). Present experimental value [1] of the muon anomalous magnetic moment $\left(a_{\mu}=(g-2)_{\mu}\right)$ indicates a $3.7 \sigma$ deviation from the SM prediction $[2-5]:^{1}$

$$
\delta a_{\mu}=a_{\mu}^{\mathrm{EXP}}-a_{\mu}^{\mathrm{SM}}=(2.706 \pm 0.726) \times 10^{-9} .
$$

On the other hand, the measurement of fine structure constant using the Cs atom gives a precise estimation of the electron anomalous magnetic moment $\left(a_{e}=(g-2)_{e}\right)$ in the SM [7] which is $2.4 \sigma$ below the experimentally measured value [8]:

$$
\delta a_{e}=a_{e}^{\mathrm{EXP}}-a_{e}^{\mathrm{SM}}=-(8.8 \pm 3.6) \times 10^{-13} .
$$

Intriguingly, the deviations are in opposite direction which makes it hard to explain in a unified way. Also, the absolute value of the deviations are not proportional to the

\footnotetext{
${ }^{1}$ See also $[6]$.
} 
square of the respective lepton mass and it is unlikely that both the anomalies share a common origin. Various suggestions have been made for the simultaneous explanation of the anomalies [9-33].

The two Higgs doublet model (2HDM) is one of the simplest extension of the SM scalar sector, where two scalar doublets are involved in the electroweak symmetry breaking. An extra Higgs doublet appears in many theories beyond the SM: supersymmetry [34], explaining the baryon asymmetry of the Universe [35, 36], or resolving the strong CP problem [37]. The presence of a relatively light neutral scalar in 2HDM does not violate the custodial symmetry [38] and thus can be consistent with the electroweak precision test [39]. It is well known that the type-X (lepton-specific) $2 \mathrm{HDM}$ with a light pseudoscalar $(A)$ can explain the muon anomaly for large $\tan \beta$ [39-48]. On the other hand, the contribution to the electron $(g-2)$ from the type-X $2 \mathrm{HDM}$ scales as $\left(m_{e} / m_{\mu}\right)^{2}$ and remains small. Moreover, the contribution has the same sign as the muon anomaly. Consequently, pure 2HDM type-X can not explain both anomalies.

Hence to explain the electron anomaly a larger contribution to $\delta a_{e}$ is needed compared to the muon case. For this, we consider the inclusion of new vector-like fermions which couple dominantly to the electron. Vector like fermions have already been studied extensively and are motivated in several BSM models, including extra dimension and grand unified theories $[49,50]$. In particular, additional new vector-like lepton (VLL) can help to explain the anomalous magnetic moment since the helicity flip which is required by the dipole transition occurs through a VLL mass insertion which is larger than the electroweak (EW) scale. The SM model with a singlet and a doublet VLL which couple exclusively to the muon has been suggested to explain $\delta a_{\mu}[51,52]$. Moreover, the VLL mass, if close to the EW scale, modifies significantly the muon Yukawa coupling which is strongly constrained by the recent measurements of $h \rightarrow \mu \mu$ by ATLAS [53] and CMS [54]. For recent studies to explain muon $(g-2)$ in $2 \mathrm{HDM}$ with vector-like lepton see $[55,56]$.

In this paper, we explore this idea in the context of the two Higgs doublet model. In particular we consider the type-X $2 \mathrm{HDM}$ where one doublet $\left(\Phi_{2}\right)$ is responsible for the masses of the quarks while the other $\left(\Phi_{1}\right)$ generates masses of the leptons. The new vectorlike leptons are supposed to couple dominantly to the electron through Yukawa couplings with $\Phi_{1}$. Since the new vector-like leptons couple to the doublet which acquires a small vacuum expectation value (vev), the corrections to the SM couplings will be small. We find that the model remains mostly unconstrained from both the precision measurements at the $Z$ pole and the oblique corrections. In this scenario, $\delta a_{e}$ is generated at one-loop owing to the mixing of the new leptons with the electron. The dominant contribution comes from the pseudo-scalar $(A)$ mediated diagram. Apart from explaining the $\delta a_{e}$, the VLL contributes also to $\delta a_{\mu}$ at two-loop which is, however, relatively small as it is suppressed by the ratio of the small chiral mass to the vector-like mass of the new leptons. Nevertheless, this additional contribution can help explain $\delta a_{\mu}$ in a larger parameter space compared to the pure type-X $2 \mathrm{HDM}$ scenario.

Our model predicts a peculiar collider signature. A pair of the vector-like leptons produced at the LHC will leave an electron-positron pair and extra Higgs bosons, especially, a light pseudo-scalar $A$ which will be boosted and decay to $\tau^{+} \tau^{-}$. Thus the over-lapping 


\begin{tabular}{|c|c|c|c|c|c|c|}
\hline Fields & $\ell_{L}$ & $e_{R}$ & $L_{L}, L_{R}$ & $E_{R}, E_{L}$ & $\Phi_{1}$ & $\Phi_{2}$ \\
\hline$Z_{2}$ Charge & + & - & + & - & - & + \\
\hline
\end{tabular}

Table 1. Table contains the $Z_{2}$ charges of leptons and scalars present in the model.

di- $\tau$ accompanied by $e^{+} e^{-}$can be searched for to probe our scenario. The novel di- $\tau$ tagger used by the ATLAS collaboration for di-Higgs searches will be useful also for the boosted $A$ search.

The paper is organized as follows: in section 2 we describe the model in detail. Then in section 3 we move on to the contribution to electron and muon anomalous magnetic moment in this model. The constraints coming from $Z$ pole precision physics and oblique parameters are discussed in section 4 . The results are described in section 5 and possible collider bounds and signals are discussed in section 6. Finally we conclude in section 7 .

\section{The model}

We consider a model with $2 \mathrm{HDM}$ (of type-X) along with vector-like leptons. That is, the SM is extended to have an additional scalar doublet, a pair of lepton doublets $L_{L, R}$ and a pair of charged lepton singlets $E_{L, R}$. The two doublets $\Phi_{1}$ and $\Phi_{2}$ have same hypercharge $\left(+\frac{1}{2}\right)$. To avoid flavor changing neutral current processes we have considered additional $\mathbb{Z}_{2}$ symmetry under which $\Phi_{1}$ is odd and $\Phi_{2}$ is even. The SM singlet leptons are odd under the $\mathbb{Z}_{2}$ symmetry which ensures that the leptons couple solely to $\Phi_{1}$. In the same spirit, we have considered that the singlets $E_{L, R}$ are odd under the $\mathbb{Z}_{2}$ symmetry. In table 1 we show charge of leptons and scalars under the $\mathbb{Z}_{2}$ symmetry. Also, we have considered the mixing of VLL with electrons only and mixing of new leptons with more than one SM family simultaneously is strongly constrained by various lepton flavor violating processes.

Relevant part of the Lagrangian containing Yukawa and mass terms(suppressing the generation index):

$$
\begin{aligned}
-\mathcal{L} \supset & y_{e} \bar{\ell}_{L} e_{R} \Phi_{1}+\lambda_{L} \bar{L}_{L} e_{R} \Phi_{1}+\lambda_{E} \bar{\ell}_{L} E_{R} \Phi_{1} \\
& +\lambda \bar{L}_{L} E_{R} \Phi_{1}+\bar{\lambda} \Phi_{1}^{\dagger} \bar{E}_{L} L_{R}+M_{L} \bar{L}_{L} L_{R}+M_{E} \bar{E}_{L} E_{R}+\text { h.c. } .
\end{aligned}
$$

Let us note that two additional mass terms $\mu_{L} \bar{\ell}_{L} L_{R}$ and $\mu_{E} \bar{E}_{L} e_{R}$ allowed in the Lagrangian can be rotated away, so that only the vector-like mass terms $\left(M_{L}\right.$ and $\left.M_{E}\right)$ and Yukawa couplings remain as free parameters.

The lepton and scalar doublets can be written as,

$$
\ell_{L}=\left(\begin{array}{c}
\nu_{e} \\
e_{L}^{-}
\end{array}\right), L_{L, R}=\left(\begin{array}{c}
L_{L, R}^{0} \\
L_{L, R}^{-}
\end{array}\right), \Phi_{1}=\left(\begin{array}{c}
\Phi_{1}^{+} \\
\Phi_{1}^{0}
\end{array}\right), \Phi_{2}=\left(\begin{array}{c}
\Phi_{2}^{+} \\
\Phi_{2}^{0}
\end{array}\right) .
$$

As usual in 2HDM, we have,

$$
\begin{aligned}
& \Phi_{1}^{0}=\frac{1}{\sqrt{2}}\left(v_{1}\left(=v c_{\beta}\right)+c_{\alpha} H-s_{\alpha} h+i c_{\beta} G^{0}-i s_{\beta} A\right) \\
& \Phi_{2}^{0}=\frac{1}{\sqrt{2}}\left(v_{2}\left(=v s_{\beta}\right)+s_{\alpha} H+c_{\alpha} h+i s_{\beta} G^{0}+i c_{\beta} A\right),
\end{aligned}
$$


where $h$ is the SM higgs boson and $H(A)$ is additional scalar(pseudo-scalar). The charged gauge eigenstates $\Phi_{1}^{+}$and $\Phi_{2}^{+}$will give rise to one charged Higgs $H^{+}$and a charged Goldstone boson. Details about the scalar sector in 2HDM can be found in [57].

After spontaneous symmetry breaking the mass matrix for charged leptons is given by:

$$
\mathcal{L}_{\text {mass }}=\left(\bar{\ell}_{L i}, \bar{L}_{L}^{-}, \bar{E}_{L}\right) \mathcal{M}_{E}\left(\begin{array}{c}
\ell_{R j} \\
L_{R}^{-} \\
E_{R}
\end{array}\right)+\text { h.c.. }
$$

Here $i, j$ denotes the light lepton generation index $(i, j=1,2,3)$ and $\mathcal{M}_{E}$ is the $5 \times 5$ mass matrix given by

$$
\mathcal{M}_{E}=\left(\begin{array}{ccc}
\frac{1}{\sqrt{2}} y_{e, i j} v_{1} & 0 & \frac{1}{\sqrt{2}} \lambda_{E_{i}} v_{1} \\
\frac{1}{\sqrt{2}} \lambda_{L_{j}} v_{1} & M_{L} & \frac{1}{\sqrt{2}} \lambda v_{1} \\
0 & \frac{1}{\sqrt{2}} \bar{\lambda} v_{1} & M_{E}
\end{array}\right),
$$

where the upper left block is the $3 \times 3$ matrix of the SM leptons. The mass matrix can be diagonalized by a bi-unitary transformation,

$$
\widetilde{U}_{L}^{\dagger} \mathcal{M}_{E} \widetilde{U}_{R}=\operatorname{diag}\left(m_{e}, m_{\mu}, m_{\tau}, m_{1}, m_{2}\right),
$$

where $m_{1}$ and $m_{2}$ denotes mass of the two new mass eigenstates $E_{1}$ and $E_{2}$ respectively. Since we are interested in the mixing of electron with the vector-like leptons, we have assumed that the $\lambda_{E_{2}}, \lambda_{E_{3}}, \lambda_{L_{2}}, \lambda_{L_{3}}=0$. From now on we will ignore the muon and tau in the charged lepton mass matrix $\mathcal{M}_{E}$ and denote $\lambda_{E_{1}\left(L_{1}\right)}=\lambda_{E(L)}$. With these simplifications we can now rewrite the eq. (2.6) as,

$$
U_{L}^{\dagger}\left(\begin{array}{ccc}
\frac{1}{\sqrt{2}} y_{e} v_{1} & 0 & \frac{1}{\sqrt{2}} \lambda_{E} v_{1} \\
\frac{1}{\sqrt{2}} \lambda_{L} v_{1} & M_{L} & \frac{1}{\sqrt{2}} \lambda v_{1} \\
0 & \frac{1}{\sqrt{2}} \bar{\lambda} v_{1} & M_{E}
\end{array}\right) U_{R}=\left(\begin{array}{ccc}
m_{e} & 0 & 0 \\
0 & m_{1} & 0 \\
0 & 0 & m_{2}
\end{array}\right) .
$$

In the above equation the diagonalization matrices $U_{L, R}$ obtained from $\widetilde{U}_{L / R}$ by removing the muon and tau entries.

In the limit

$$
\frac{\lambda_{L} v_{1}}{\sqrt{2}}, \frac{\lambda_{E} v_{1}}{\sqrt{2}}, \frac{\bar{\lambda} v_{1}}{\sqrt{2}}, \frac{\lambda v_{1}}{\sqrt{2}} \ll M_{E}, M_{L}
$$

approximate analytic formulas for diagonalization matrices can be obtained [52, 58],

$$
U_{L}=\left(\begin{array}{ccc}
1-\frac{v_{1}^{2}}{2} \frac{\lambda_{E}^{2}}{2 M_{E}^{2}} & -\frac{v_{1}^{2}}{2}\left(\frac{\lambda_{E}}{M_{L}} \frac{\bar{\lambda} M_{E}+\lambda M_{L}}{M_{E}^{2}-M_{L}^{2}}-\frac{y_{e} \lambda_{L}}{M_{L}^{2}}\right) & \frac{v_{1}}{\sqrt{2}} \frac{\lambda_{E}}{M_{E}} \\
\frac{v_{1}^{2}\left(\bar{\lambda} \lambda_{E} M_{L}-y_{e} \lambda_{L} M_{E}\right)}{2 M_{L}^{2} M_{E}} & \cos \theta_{L} & -\sin \theta_{L} \\
-\frac{v_{1}}{\sqrt{2}} \frac{\lambda_{E}}{M_{E}} & \sin \theta_{L} & \cos \theta_{L}
\end{array}\right),
$$

and

$$
U_{R}=\left(\begin{array}{ccc}
1-\frac{v_{1}^{2}}{2} \frac{\lambda_{L}^{2}}{2 M_{L}^{2}} & \frac{v_{1}}{\sqrt{2}} \frac{\lambda_{L}}{M_{L}} & \frac{v_{1}^{2}}{2}\left(\frac{\lambda_{L}}{M_{E}} \frac{\bar{\lambda} M_{L}+\lambda M_{E}}{M_{E}^{2}-M_{L}^{2}}+\frac{y_{e} \lambda_{E}}{M_{E}^{2}}\right) \\
-\frac{v_{1}}{\sqrt{2}} \frac{\lambda_{L}}{M_{L}} & \cos \theta_{R} & -\sin \theta_{R} \\
\frac{v_{1}^{2}}{2} \frac{\left(\lambda_{L} \bar{\lambda} M_{E}-y_{e} \lambda_{E} M_{L}\right)}{M_{L} M_{E}^{2}} & \sin \theta_{R} & \cos \theta_{R}
\end{array}\right),
$$


where the $2 \times 2$ matrix $R\left(\theta_{L / R}\right)$ diagonalizes the 2-3 block of $\mathcal{M}_{E} \mathcal{M}_{E}^{\dagger}$ and $\mathcal{M}_{E}^{\dagger} \mathcal{M}_{E}$ respectively.

\subsection{Couplings of the scalars and fermions}

From eqs. (2.1) and (2.3) we can write the coupling of the electron and the new vector-like fermions with the pseudo-scalar as

$$
\begin{aligned}
\mathcal{L}_{A \chi_{a} \chi_{b}} & =-i \frac{1}{\sqrt{2}} s_{\beta} A\left(e_{L}, L_{L}^{-}, E_{L}\right)\left(\begin{array}{ccc}
y_{e} & 0 & \lambda_{E} \\
\lambda_{L} & 0 & \lambda \\
0 & \bar{\lambda} & 0
\end{array}\right)\left(\begin{array}{c}
e_{R} \\
L_{R}^{-} \\
E_{R}
\end{array}\right)+h . c . \\
& =-i \tan \beta \frac{\xi_{a b}}{v} A \bar{\chi}_{L a} \chi_{R b}+h . c .
\end{aligned}
$$

where $\chi_{L / R}=\left(e, E_{1}, E_{2}\right)_{L / R}$ are the mass eigenstates obtained from diagonalization by $U_{L / R}$ and the mass matrix $\xi_{a b}$ is given by

$$
\xi_{a b}=\left[\operatorname{diag}\left(m_{e}, m_{1}, m_{2}\right)-U_{L}^{\dagger} \operatorname{diag}\left(0, M_{L}, M_{E}\right) U_{R}\right]_{a b} .
$$

Similarly, one obtains the Yukawa couplings of the scalars $H$ and $h$ as

$$
\mathcal{L} \supset \frac{c_{\alpha}}{c_{\beta}} \frac{1}{v} \xi_{a b} H \bar{\chi}_{L a} \chi_{R b}-\frac{s_{\alpha}}{c_{\beta}} \frac{1}{v} \xi_{a b} h \bar{\chi}_{L a} \chi_{R b}+\text { h.c. }
$$

From the expression of $\xi_{a b}$ it is evident that if there are no new fermion fields then $\xi_{a b}$ reduces to the mass of the lepton $\left(\xi_{11}=m_{e}\right)$ and the standard 2HDM Yukawa couplings are recovered:

$$
\mathcal{L} \supset-i y_{\ell}^{A}\left(\frac{m_{\ell}}{v}\right) A \bar{\ell} \gamma_{5} \ell+y_{\ell}^{H}\left(\frac{m_{\ell}}{v}\right) H \bar{\ell} \ell+y_{\ell}^{h}\left(\frac{m_{\ell}}{v}\right) h \bar{\ell} \ell
$$

with the prefactors $y_{l}^{A, H, h}$ specific in the type-X model:

$$
y_{\ell}^{A}=\tan \beta, \quad y_{\ell}^{H}=\frac{c_{\alpha}}{c_{\beta}} \quad \text { and } \quad y_{\ell}^{h}=\frac{-s_{\alpha}}{c_{\beta}} .
$$

To denote coupling of $A$ and $H$ with electron and the vector-like leptons, we will use convention of eq. (2.12) and (2.14). On the other hand, for muon and tau we will use the notation of eq. (2.15). This is to emphasize the fact that only the electron coupling is affected by VLLs.

\subsection{Couplings to gauge bosons}

Apart from the pseudo-scalar mediated diagram, there will be an additional diagram for $\delta a_{e}$ mediated by the SM gauge bosons. Here we give explicit expressions relevant for the computation of $\delta a_{e}$. The couplings of $Z$ boson with electron and the heavy fermions is given by,

$$
\begin{aligned}
g_{L}^{Z e E_{1}} & =\frac{g}{2 \cos \theta_{W}}\left(U_{L}^{\dagger}\right)_{13}\left(U_{L}\right)_{32}, & g_{L}^{Z} e E_{2} & =\frac{g}{2 \cos \theta_{W}}\left(U_{L}^{\dagger}\right)_{13}\left(U_{L}\right)_{33} \\
g_{R}^{Z e E_{1}} & =-\frac{g}{2 \cos \theta_{W}}\left(U_{R}^{\dagger}\right)_{12}\left(U_{R}\right)_{22}, & g_{R}^{Z e E_{2}} & =-\frac{g}{2 \cos \theta_{W}}\left(U_{R}^{\dagger}\right)_{12}\left(U_{R}\right)_{23}
\end{aligned}
$$



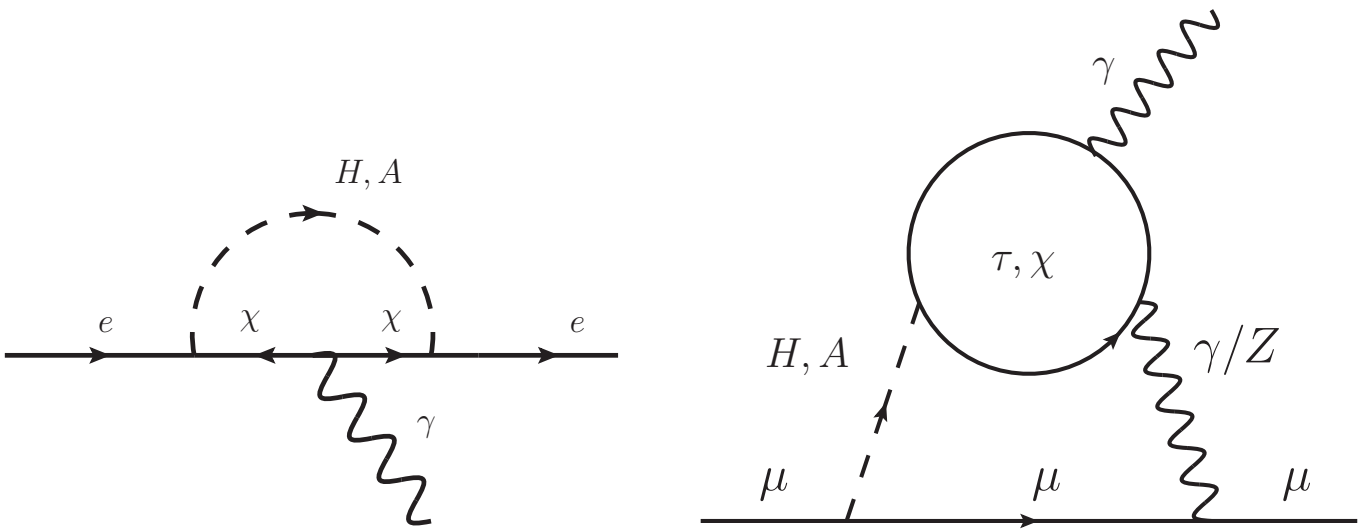

Figure 1. Left figure shows electron $(g-2)$ dominant contribution from the chirality flip of the heavy leptons and the right figure is a representative two-loop Barr-Zee diagram which explain the muon $(g-2)$. Here $\chi$ denotes the vector-like leptons.

Similarly, the coupling of the $W$ boson with electron and the neutral heavy lepton $(N)$ is given by

$$
g_{L}^{W N}=\frac{g}{\sqrt{2}}\left(U_{L}\right)_{21}, \quad g_{R}^{W N}=\frac{g}{\sqrt{2}}\left(U_{R}\right)_{21}
$$

Note that all the couplings are proportional to the $v_{1}$ and hence the gauge boson couplings are always much smaller than the $A-V L L$-electron couplings. This is important to satisfy the precision constraints.

\section{Electron and muon anomalous magnetic moment}

\subsection{Electron $(g-2)$}

The dominant contribution to the anomalous magnetic moment will come from diagrams with a chiral flip of the VLL in the loop as shown in figure 1. The contribution from the pseudo-scalar $A$ in the loop is given by [59],

$$
\delta a_{e}^{A}=-\frac{m_{e}^{2}}{8 \pi^{2} m_{A}^{2}}\left(\frac{\tan \beta}{v}\right)^{2} \sum_{i=1,2} \xi_{1 i} \xi_{i 1} I_{-}\left(\frac{m_{e}^{2}}{m_{A}^{2}}, \frac{m_{i}^{2}}{m_{A}^{2}}\right),
$$

and the heavy neutral Higgs $H$ mediated diagram gives,

$$
\delta a_{e}^{H}=-\frac{m_{e}^{2}}{8 \pi^{2} m_{H}^{2}}\left(\frac{c_{\alpha}}{c_{\beta}} \frac{1}{v}\right)^{2} \sum_{i=1,2} \xi_{1 i} \xi_{i 1} I_{+}\left(\frac{m_{e}^{2}}{m_{H}^{2}}, \frac{m_{i}^{2}}{m_{H}^{2}}\right) .
$$

The loop functions are,

$$
I_{ \pm}(a, b)=\int_{0}^{1} d x \frac{x^{2}(1-x \pm \epsilon)}{(1-x)(1-x a)+x b} \quad \text { where } \epsilon=\frac{m_{i}}{m_{e}} .
$$

There are also diagrams with the charged Higgs boson and heavy neutral lepton in the loop. However, their contribution becomes negligible due to lack of a chiral enhancement. 
The contribution from the $Z$ diagram is given by

$$
\delta a_{e}^{Z}=-\frac{m_{e}}{8 \pi^{2} m_{Z}^{2}} \sum_{i=1,2}\left[\left(g_{L}^{Z e E_{i}} g_{R}^{Z e E_{i}}\right) m_{i} G_{Z}\left(x_{Z, i}\right)\right]
$$

where $x_{Z, i}=\left(m_{i} / M_{Z}\right)^{2}$, the couplings are expressed in eq. (2.17) and the loop function is as follows:

$$
G_{Z}(x)=\frac{x^{3}+3 x-6 x \ln (x)-4}{2(1-x)^{3}} .
$$

The $W$ mediated diagram yields,

$$
\delta a_{e}^{W}=-\frac{m_{e}}{16 \pi^{2} m_{W}^{2}}\left[\left(g_{L}^{W N} g_{R}^{W N}\right) M_{L} G_{W}\left(x_{W}\right)\right],
$$

where $x_{W}=\left(M_{L} / M_{W}\right)^{2}$. The couplings are written in eq. (2.18) and the loop function is:

$$
G_{\mathrm{W}}(x)=-\frac{x^{3}-12 x^{2}+15 x+6 x^{2} \ln (x)-4}{(1-x)^{3}} .
$$

Contribution from gauge boson diagrams is much smaller than the pseudo-scalar one since this couplings are $\tan \beta$ suppressed compared to $A$.

Please note that we have not included the diagrams which contain only the SM particles as they are in principle included in the EW contribution from the Standard Model. These pure SM diagrams do not contribute substantially due to lack of any chiral enhancement.

\subsection{Muon $(g-2)$}

The vector-like leptons do not couple to muons, and there are no new VLL loops for muon $(g-2)$ at one loop. The one-loop diagrams are subdominant compared to the two-loop Barr-Zee (BZ) diagrams with heavy fermions ( $\tau$ or VLL) in the loop as shown in right panel of figure 1 . The new vector-like leptons will give an additional contribution to the muon $(g-2)$. The dominant contribution reads as

$$
\delta a_{\mu}^{A}=\frac{\alpha_{e m}}{4 \pi^{3}} \frac{m_{\mu}^{2}}{v^{2}}\left[\sum_{i=1,2} Q_{E_{i}}^{2} y_{\mu}^{A} \frac{v}{m_{i}} g_{A E_{i} E_{i}} \mathcal{F}\left(\frac{m_{i}^{2}}{m_{A}^{2}}\right)+Q_{\tau}^{2} y_{\mu}^{A} y_{\tau}^{A} \mathcal{F}\left(\frac{m_{\tau}^{2}}{m_{A}^{2}}\right)\right] .
$$

Relevant contribution coming from the heavy neutral higgs is given by

$$
\delta a_{\mu}^{H}=\frac{\alpha_{e m}}{4 \pi^{3}} \frac{m_{\mu}^{2}}{v^{2}}\left[\sum_{i=1,2} Q_{E_{i}}^{2} y_{\mu}^{H} \frac{v}{m_{i}} g_{H E_{i} E_{i}} \mathcal{G}\left(\frac{m_{i}^{2}}{m_{H}^{2}}\right)+Q_{\tau}^{2} y_{\mu}^{H} y_{\tau}^{H} \mathcal{G}\left(\frac{m_{\tau}^{2}}{m_{H}^{2}}\right)\right] .
$$

The loop functions are,

$$
\begin{aligned}
\mathcal{F}(x) & =\frac{x}{2} \int_{0}^{1} d y \frac{1}{y(1-y)-x} \ln \left(\frac{y(1-y)}{x}\right), \\
\mathcal{G}(x) & =\frac{x}{2} \int_{0}^{1} d y \frac{2 y(1-y)-1}{y(1-y)-x} \ln \left(\frac{y(1-y)}{x}\right) .
\end{aligned}
$$


The factors $g_{A E_{i} E_{i}}$ and $g_{H E_{i} E_{i}}$ can be calculated from eq. (2.12) and (2.14):

$$
g_{A E_{i} E_{i}}=\tan \beta \frac{1}{v} \xi_{i i} \quad \text { and } \quad g_{H E_{i} E_{i}}=\frac{c_{\alpha}}{c_{\beta}} \frac{1}{v} \xi_{i i} .
$$

The factors $y_{\mu / \tau}^{A / H}$ are defined in eq. (2.15). Contribution coming from the tau-loop is shown in the second term of eq. (3.8) and (3.9) and is same as in type-X 2HDM [39-48]. As discussed previously, we have used 2HDM conventions for muon and tau loop because only electron coupling is affected by VLLs.

The Yukawa modifiers $y_{\mu}^{H}$ and $y_{\tau}^{H}$ has the same value $\frac{c_{\alpha}}{c_{\beta}}$ which goes as $\tan \beta$ in the limit $\sin (\beta-\alpha) \simeq 1$ as indicated by the Higgs measurements at the LHC [60]. When the heavy Higgs is lighter than the vector like particle in the loop then the contribution is not suppressed by the heavy Higgs mass and nearly comparable to the light pseudoscalar contribution. Moreover, the contribution from $H$ mediated diagram is negative and partially cancels the $A$ mediated diagram. Hence, the overall effect of the VLLs in the muon $g-2$ is inadequate to enhance allowed parameter space significantly compare to the vanilla type-X 2HDM. On the other hand, when the heavy $\mathrm{CP}$ even Higgs is heavier than the vector-like lepton, the cancellation among $A$ and $H$ mediated process is relatively small. In the next section we will quantify these statements.

Like the electron case, here also the possible charged Higgs diagrams are omitted as they give very small contribution $(\leq 1-2 \%)$. The expression for $\delta a_{\mu}$ originating from a charged Higgs diagram is given in [42] and the relevant form factor for a $H^{ \pm}$decay to $W^{ \pm} \gamma$ via a vector-like fermion loop is given in [61].

\section{Constraints from precision observables}

\subsection{Constraints from the $Z$ pole measurements}

We have discussed in the previous section that the mixing of the new leptons with the electron is important to explain $\delta a_{e}$. However, the mixing modifies the coupling of the electron to the gauge bosons, and the precision measurement at the $Z$ pole [62] can constrain the mixing. There are three dimension- 6 effective operators which can directly modify the lepton gauge coupling,

$$
\mathcal{L}_{\text {eff }}=\frac{1}{\Lambda^{2}}\left(C_{\phi \ell}^{1, i j} \mathcal{O}_{\phi \ell}^{1, i j}+C_{\phi \ell}^{3, i j} \mathcal{O}_{\phi \ell}^{3, i j}+C_{\phi e}^{i j} \mathcal{O}_{\phi e}^{i j}\right)
$$

where,

$$
\begin{aligned}
\mathcal{O}_{\phi \ell}^{1, i j} & =i\left(\phi^{\dagger} \overleftrightarrow{D_{\mu}} \phi\right)\left(\bar{\ell}_{L}^{i} \gamma^{\mu} \ell_{L}^{j}\right), \quad \mathcal{O}_{\phi \ell}^{3, i j}=i\left(\phi^{\dagger} \overleftrightarrow{D_{\mu} a} \phi\right)\left(\bar{\ell}_{L}^{i} \gamma^{\mu} \tau^{a} \ell_{L}^{j}\right) \\
\mathcal{O}_{\phi e}^{i j} & =i\left(\phi^{\dagger} \overleftrightarrow{D_{\mu}} \phi\right)\left(\bar{e}_{R}^{i} \gamma^{\mu} e_{R}^{j}\right)
\end{aligned}
$$

For a model with a doublet VLL (which couples to the SM singlet) and a singlet VLL (couples to the SM lepton doublet) we have the following Wilson coefficients,

$$
\frac{C_{\phi \ell}^{1, i j}}{\Lambda^{2}}=\frac{C_{\phi \ell}^{3, i j}}{\Lambda^{2}}=-\frac{\lambda_{E}^{2}}{4 M_{E}^{2}} \quad \text { and } \quad \frac{C_{\phi e}^{i j}}{\Lambda^{2}}=+\frac{\lambda_{L}^{2}}{2 M_{L}^{2}}
$$


In our scenario the leptons as well as the VLLs couple exclusively to $\Phi_{1}$. Consequently, the global electroweak fit for the vector like leptons gives the following limit [51,63]:

$$
\frac{v_{1}\left|\lambda_{E}\right|}{M_{E}} \leq 0.04 \quad \text { and } \quad \frac{v_{1}\left|\lambda_{L}\right|}{M_{L}} \leq 0.02
$$

We will satisfy this limit throughout.

\subsection{Constraints from oblique corrections}

So far we have seen that the Yukawa couplings which induce mixing of the SM leptons with the VLLs can be constrained by the precision observables. However, the $Z$ pole observables can not constrain the coupling $\lambda$ and $\bar{\lambda}$ which mixes the VLLs among themselves. Interestingly, the mass eigenstates of the heavy charged leptons depend on these couplings, and they can induce a mass gap between the neutral and the charged component of the doublet. This can give correction to oblique $T$ parameter $[64,65]$ and can be constrained. Contribution to the $T$ parameter from the VLL is given by [66],

$$
\begin{aligned}
\Delta T= & \frac{1}{16 \pi s_{W}^{2} c_{W}^{2}}\left[\left(c_{L}^{2}+c_{R}^{2}\right) \theta_{+}\left(y_{1}, y_{L}\right)+\left(s_{L}^{2}+s_{R}^{2}\right) \theta_{+}\left(y_{2}, y_{L}\right)+2 c_{L} c_{R} \theta_{-}\left(y_{1}, y_{L}\right)\right. \\
& \left.\left.+2 s_{L} s_{R} \theta_{-}\left(y_{2}, y_{L}\right)\right)-\left(s_{L}^{2} s_{R}^{2}+c_{L}^{2} c_{R}^{2}\right) \theta_{+}\left(y_{1}, y_{2}\right)-2 s_{L} s_{R} c_{L} c_{R} \theta_{-}\left(y_{1}, y_{2}\right)\right] .
\end{aligned}
$$

Where $y_{i}=M_{i}^{2} / M_{Z}^{2}$ and for the heavy neutral particle we denote its mass as $M_{L}$ disregarding the small radiative correction. The mixing angle $c_{L / R} \equiv \cos \theta_{L / R}$ etc., are components of the unitary matrices shown in eq. (2.9) and (2.10). The functions $\theta_{ \pm}$are,

$$
\begin{aligned}
& \theta_{+}\left(y_{1}, y_{2}\right)=y_{1}+y_{2}-\frac{2 y_{1} y_{2}}{y_{1}-y_{2}} \ln \frac{y_{1}}{y_{2}} \\
& \theta_{-}\left(y_{1}, y_{2}\right)=2 \sqrt{y_{1} y_{2}}\left(\frac{y_{1}+y_{2}}{y_{1}-y_{2}} \ln \frac{y_{1}}{y_{2}}-2\right) .
\end{aligned}
$$

We have scanned the parameters in our model as shown in table 2 and found that all points satisfy the constraints. In our scenario, the mass difference originates from the vev of $\Phi_{1}$, which is in general small for large $\tan \beta$. Hence, the constraints from the $T$ parameter can be easily satisfied.

Apart from the vector-like leptons, the scalar sector in 2HDM can also contribute to $T$ parameter. However, it has been shown that $[38,39]$ the oblique corrections from the scalar sector of $2 \mathrm{HDM}$ can be minimized by making the charged Higgs degenerate with the heavy scalar or the pseudo-scalar. We will use this mass spectrum in our analysis.

\section{Results and discussion}

In this section, we will present the numerical results for $\delta a_{e}$ and $\delta a_{\mu}$, which satisfy the precision constraints. We have scanned the available parameter space shown in table 2 . The Yukawa couplings $\lambda$ and $\bar{\lambda}$ are relatively unconstrained due to small vev of $\Phi_{1}$ as discussed in section 4.2. Hence we have scanned the full range allowed by perturbativity. 


\begin{tabular}{|c|c|c|c|c|c|c|}
\hline Parameters & $v_{1} \frac{\left|\lambda_{L / E}\right|}{M_{L / E}}$ & $\lambda, \bar{\lambda}$ & $M_{L}(\mathrm{GeV})$ & $\Delta M=\frac{M_{E}-M_{L}}{M_{E}+M_{L}}$ & $M_{A}(\mathrm{GeV})$ & $\tan \beta$ \\
\hline Range & $\left(10^{-1}, 10^{-5}\right)$ & $(-\sqrt{4 \pi}, \sqrt{4 \pi})$ & $(500,1000)$ & $(0.01,0.10)$ & $(30,150)$ & $(30,100)$ \\
\hline
\end{tabular}

Table 2. Range of the scanned parameters.

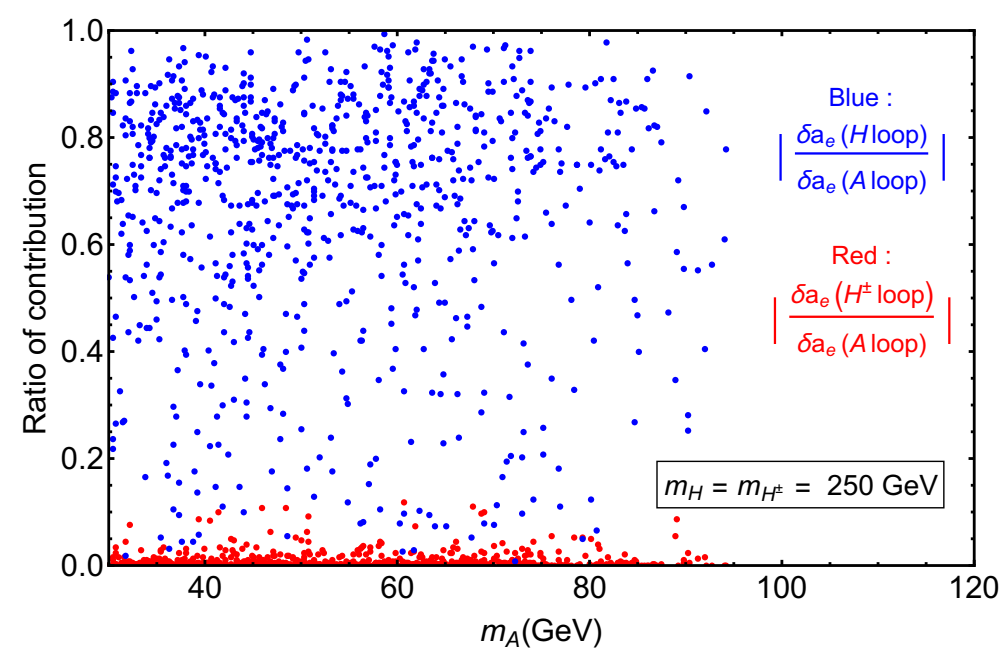

Figure 2. Electron $(g-2)$ contribution coming from the heavy neutral and charged Higgs mediated diagram relative to the pseudo-scalar mediated diagram is shown here. We have plotted the absolute value and the contribution coming from $H\left(H^{ \pm}\right)$mediated diagram is opposite to(same as) the $A$ mediated diagram.

The BZ diagram contribution to $\delta a_{\mu}$ coming from VLL loop also depends on the singletdoublet mixing in the VLL sector as only diagonal couplings from $A(H) \chi_{i} \chi_{j}$ appears in the $H$ or $A$ mediated loops. If the singlet and doublet mass parameter, i.e. $M_{E}$ and $M_{L}$ are well separated then the mixing will be small and $A(H) \chi_{i} \chi_{j}$ coupling will be mostly off-diagonal and contribution from VLL loop for $\delta a_{\mu}$ will vanish. Hence, for our analysis, we have assumed that the mass difference between the vector-like masses is small and varied the parameter $\Delta M\left(=\frac{M_{E}-M_{L}}{M_{E}+M_{L}}\right)$ in a small range which allows large mixing. For the same reason, the dominant contribution to $\delta a_{\mu}$ comes when $\lambda$ or $\bar{\lambda}$ is relatively large.

\subsection{Results for electron $(g-2)$}

The expressions of new physics contributions for $\delta a_{e}$ is given in section 3.1. The dominant contribution comes from the pseudo-scalar and heavy Higgs mediated diagram due to $\tan \beta$ enhancement. The contributions from the gauge boson mediated diagrams are at the percent level or below.

Since the $H$ and $A$ contributions have opposite sign, they will cancel partially. In figure 2 we have plotted absolute value of contribution coming from the $H$ and $H^{ \pm}$mediated diagram relative to the $A$ mediated diagram as a function of the $A$ mass. Here we set the heavy Higgs and the charged Higgs mass at $250 \mathrm{GeV}$ for illustration. The contribution from $H$ is not suppressed much and is a bit smaller than the $A$ contribution. The suppression 


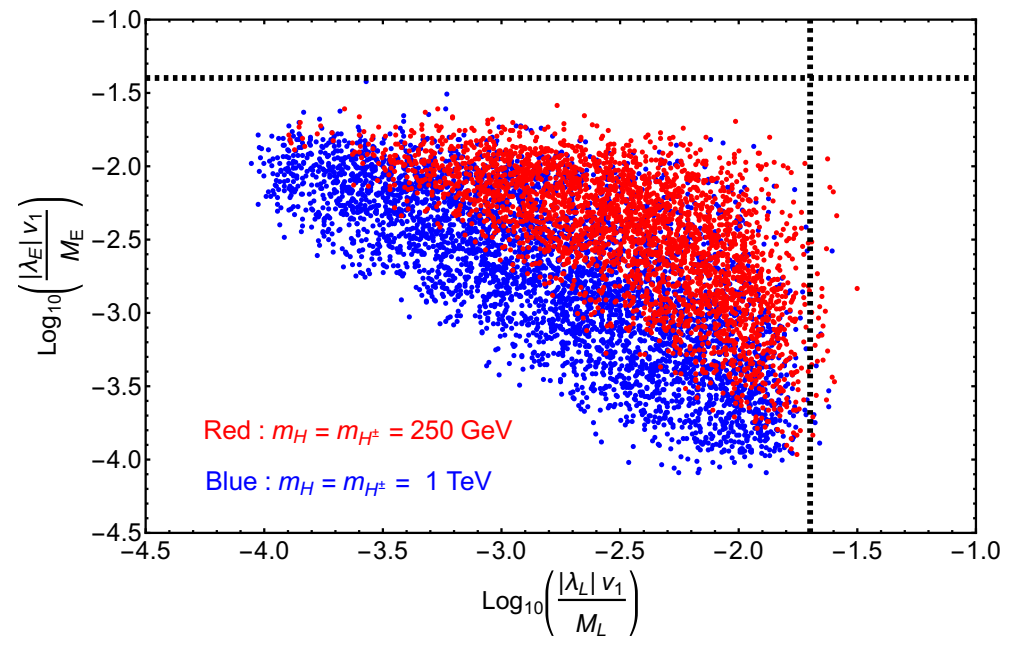

Figure 3. The parameter space in the $\lambda_{L}-\lambda_{E}$ plane which can explain the $\delta a_{e}$ anomaly at $2 \sigma$. All the other parameters are varied as shown in table 2 . The black dashed lines are constraints coming from $Z$ pole observables.

will be substantial for $m_{H} \gg M_{L, E}$. On the other hand, the charge Higgs contribution remains low having no chiral enhancement.

In figure 3 we have displayed the parameter space in $\lambda_{L}-\lambda_{E}$ plane which can explain the electron $(g-2)$ anomaly. The red and blue points illustrate the allowed space for the heavy scalar mass $250 \mathrm{GeV}$ and $1 \mathrm{TeV}$, respectively. For higher mass, the contribution from $H$ diagram decreases and relatively small values of $\lambda_{L}\left(\lambda_{E}\right)$ can explain $\delta a_{e}$. The contribution to $\delta a_{e}$ dominantly comes from the helicity flipping terms of the vector-like lepton mass and the contribution is proportional to the factor $\lambda_{L} \lambda_{E} \bar{\lambda} v_{1}^{2} /\left(M_{L} M_{E}\right)$. This explains the lower bound and correlation among $\lambda_{L}$ and $\lambda_{E}$. The black dashed lines show constraints coming from the $Z$ pole observations as discussed in section 4.1. Most of the parameter space remains unconstrained since the limit coming from $Z$ pole observation is weak. Apart from precision measurements, perturbativity of the couplings $\lambda_{L / E}(\leq \sqrt{4 \pi})$ sets an upper limit on $\frac{\lambda_{L / E} v_{1}}{M_{L / E}}$. The maximum possible value is -1.36 for $\lambda_{L / E}=\sqrt{4 \pi}, \tan \beta=40$ and $M_{L / E}=500 \mathrm{GeV}$. Hence, there are no points above the horizontal dotted line which is at -1.40 from eq. (4.4). Also, from figure 4, it is evident that very few points are allowed for small $\tan \beta$ which satisfy $\delta a_{\mu}$. Consequently, there are very few points close to (beyond) the horizontal (vertical) line in figure 3 . As $\tan \beta$ increases, $v_{1}$ decreases, which eventually pushes the points towards lower values. Similarly, higher values of $M_{L / E}$ will drive the points towards lower value.

We would like to mention that the helicity flipping terms of the vector-like lepton mass and mixing of electron with the vector-like leptons give rise to new physics contribution in electron mass $m_{e}^{N P} \sim \lambda_{L} \lambda_{E} \bar{\lambda} v_{1}^{2} /\left(M_{L} M_{E}\right)$. This contribution comes out to be less than $10 \%$ and do not alter electron Yukawa coupling or the $h \rightarrow e e$ rates which appears in SM+VLL models [11]. 


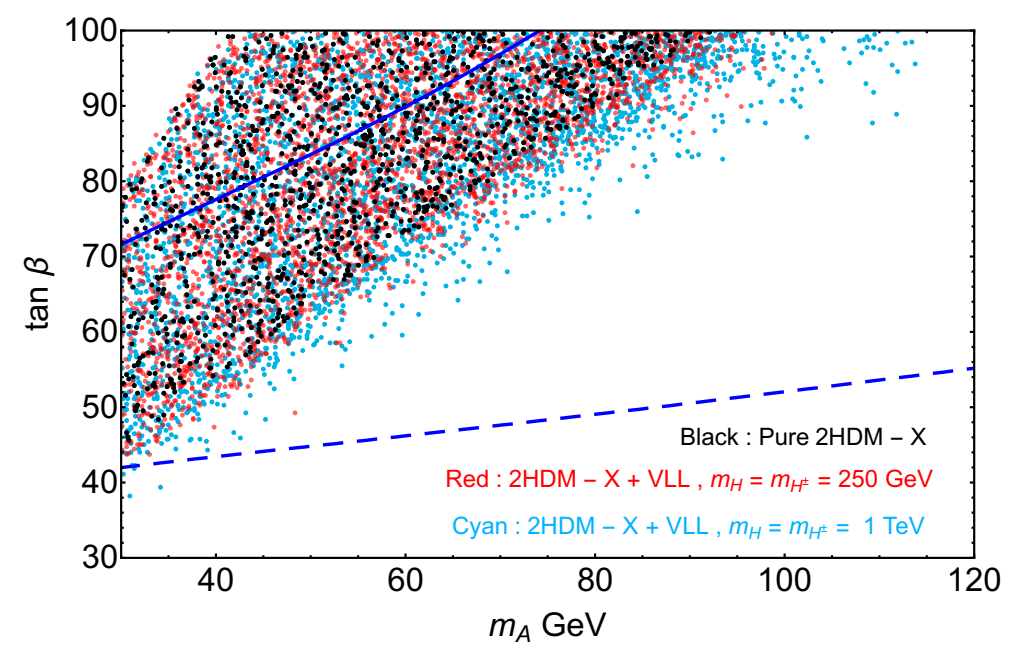

Figure 4. The parameter space in the $m_{A}-\tan \beta$ plane which can explain muon anomalous magnetic moment at $2 \sigma$. The blue solid and dashed lines depict restriction coming from the measurement of $Z \rightarrow \ell \ell$ decay.

\section{$5.2 \quad$ Results for muon $(g-2)$}

The muon $(g-2)$ anomaly can be explained in the type-X 2HDM model with a light pseudoscalar when $\tan \beta$ is large. The dominant contribution comes from the two-loop Barr-Zee diagram with tau loop. In the present model, in addition to the pure 2HDM contribution, there will be contribution coming from BZ diagram with vector-like leptons in the loop. In figure 4 we showed the parameter space in $m_{A}-\tan \beta$ plane which can explain muon anomaly at $2 \sigma$. The black colored points in figure 4 show the parameter space where muon anomaly can be explained in pure type-X 2HDM. In our model additional positive contribution for $\delta a_{\mu}$ comes from the pseudo-scalar-VLL loop, whereas the heavy scalar-VLL loop contributes negatively. Hence, the parameter space depends on both pseudo-scalar mass and heavy scalar mass. For a very heavy $H(1 \mathrm{TeV})$ the negative contribution is moderate and larger parameter space can explain $\delta a_{\mu}$ as shown by cyan colored points. On the other hand, when $H$ is relatively light the cancellation among the $A$ and $H$ mediated diagram is large resulting a marginal improvement over the pure type-X parameter space. The allowed parameter space for $m_{H / H^{ \pm}}=250 \mathrm{GeV}$ is shown in red points.

To illustrate the effect of a heavy scalar in both electron and muon $(g-2)$, we have chosen also $m_{H / H^{ \pm}}=1 \mathrm{TeV}$ in figure 4 , which is however tightly constrained by the observation of lepton universality in $Z$ and tau lepton decays [45, 47]. We have shown the $2 \sigma$ limits in blue solid $\left(m_{H / H^{ \pm}}=250 \mathrm{GeV}\right)$ and blue dashed $\left(m_{H / H^{ \pm}}=1 \mathrm{TeV}\right)$ curves coming from the observation of $Z \rightarrow \ell \ell$ decays. The limit becomes stronger for larger hierarchy between the heavy scalars $\left(H / H^{ \pm}\right)$and the light pseudoscalar and excludes most of the allowed parameter space for $m_{H / H^{ \pm}}=1 \mathrm{TeV}$. Here we have not shown the limits from $\tau$ decays as they are much weaker.

The contribution coming from the VLL does not alter the parameter space significantly. The ratio of the contributions coming from the VLL loop and tau loop is shown in figure 5 


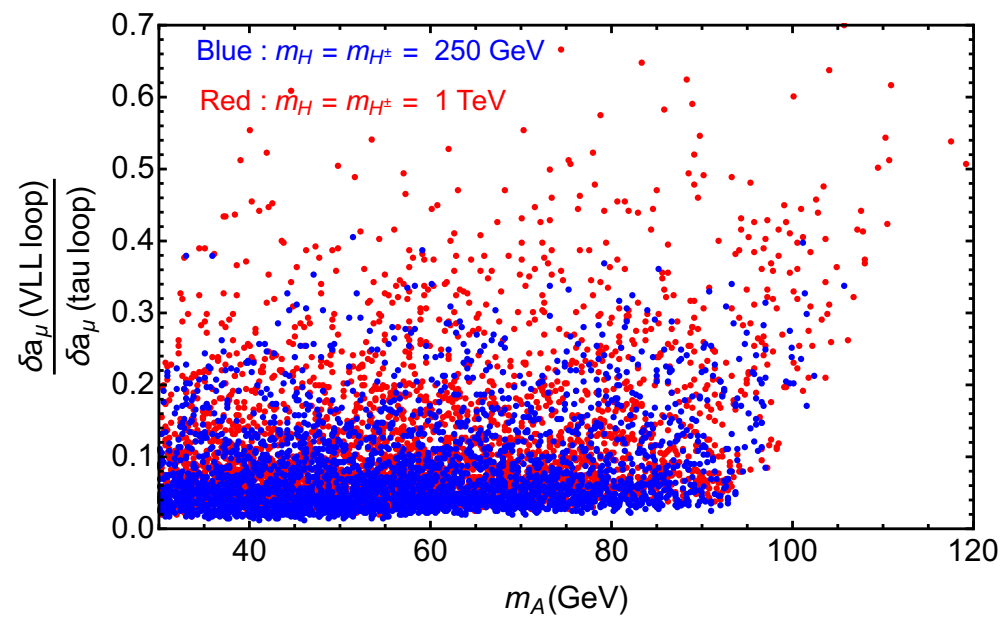

Figure 5. Ratio of the Barr-Zee diagram contribution with VLL and $\tau$ in the loop towards muon $(g-2)$ as a function of pseudo-scalar mass.

as a function of the pseudo-scalar mass. The red and blue colored points in figure 5 represent the ratio for two different values of heavy scalar mass. For heavier scalar $H$ the contribution due to vector-like lepton is larger compared to relatively lighter $H$. When the pseudo-scalar mass is relatively small the tau loop is enough to explain the muon anomaly. However, for $m_{A} \sim 100 \mathrm{GeV}$ the VLL loop need to contribute substantially. The VLL loop contribution remain small compared to tau loop since the mass insertion in the fermion loop in BZ diagram gives the contribution proportional to the ratio of chiral mass and vector-like mass $\left(\frac{\lambda / \bar{\lambda} v_{1}}{M_{L / E}}\right)$.

\section{Collider phenomenology}

In the scenario where only a VLL is added to the SM, the charged component of a doublet VLL decays equally to a lepton and the $Z /$ Higgs boson, whereas the neutral component decays to $\ell W$. The branching fraction of a heavy singlet charged VLL decay into $W \ell, Z \ell$ and $h \ell$ channel is 2:1:1. The search for a vector-like $\tau^{\prime}$ doublet at the LHC excludes $\tau^{\prime}$ up to $790 \mathrm{GeV}$ [67] using $77 \mathrm{fb}^{-1}$ data. The strongest limit comes from the di-lepton+tau-jet signal which alone excludes $\tau^{\prime}$ up to $740 \mathrm{GeV}$. However, these limits do not directly apply to our model as the decay channels are completely different as we will discuss now.

In our model, the vector-like leptons couple to the leptophilic doublet $\Phi_{1}$ and consequently the VLL decays to the new scalars $A, H$ and $H^{ \pm}$. The coupling of the vector-like leptons to the gauge bosons and light leptons is $\tan \beta$ suppressed and is negligible. Since the doublet vector-like leptons $\left(\equiv\left(L^{0}, L^{-}\right)^{T}\right)$ can be produced through the gauge interaction, the dominant production will be via the $W$ boson and we will get the following decay chain:

$$
p p \rightarrow W^{*+} \rightarrow L^{0} L^{+} \rightarrow\left(H^{+} e^{-}\right)\left(H / A e^{+}\right) \rightarrow e^{+} e^{-} H^{+} H / A .
$$

The scalars $H^{ \pm}(H)$ decays to $W^{ \pm} A(Z A)$ and $\tau \nu(\tau \tau)$ depending on $\tan \beta$ and mass of the pseudo-scalar [68-70]. The light pseudo-scalar $(A)$ decays to a pair of taus since coupling to 
other leptons is Yukawa suppressed. Hence, depending on the decay channel of the scalars, a plethora of $\tau$ rich signals along with a pair of high $p_{T}$ electrons is possible in this model. Apart from the above production channel the charged component of the doublet, as well as the singlet, charged vector-like lepton can be pair produced via the $Z$ boson. However, the production cross-section will be much smaller $(\sim 10 \%)$ than the $W$ boson channel.

Since the VLL is heavy and decays to a light $A$, our model predicts a very unique signature at the LHC. In the decay $L^{+} \rightarrow e^{+} A$ the transverse momentum for $A$ goes as $p_{T}(A) \sim \frac{m_{L^{+}}^{2}-m_{A}^{2}}{2 m_{L^{+}}}$and the decay products of $A$ will lie within the cone $2 m_{A} / p_{T}(A)$. Hence, when the vector-like lepton is very heavy the light $A$ will be highly boosted, and the tau jet pair will appear as a single merged jet as the separation will be smaller than $\Delta R=0.5$ which is required for tau-jet isolation. Signal of this kind of merged tau pair can be searched by looking for a lepton in the close proximity of a small radius tau jet. Also the 'di- $\tau$ tagger' [71] used by the ATLAS collaboration for boosted Higgs searches can be useful to look for a leptophilic extended Higgs sector. Hence a dedicated collider study is necessary to look for the VLL within 2HDM scenario and is beyond the scope of this paper. It is also remarkable that such a light pseudo-scalar can be readily probed by future linear colliders through the Yukawa process [72].

\section{Conclusion}

In conclusion, the type-X $2 \mathrm{HDM}$ extended with vector-like lepton doublet and singlet is suggested to explain both the electron and the muon $(g-2)$ anomaly. For this, the presence of a light pseudo-scalar is crucial to give a sizable positive contribution to the muon $(g-2)$ at two-loop and an appropriate negative contribution to the electron $(g-2)$ with the VLL at one-loop. These features of the model can be tested at the LHC by looking for a merged $\tau^{+} \tau^{-}$pair accompanied by a pair of $e^{+} e^{-}$. The constraints coming from the precision observables can be easily satisfied since the new VLL particles couples to the doublet $\Phi_{1}$ which gets a small vev.

\section{Acknowledgments}

EJC and TM are supported by KIAS Individual Grants PG012504 (EJC) and PG073501 (TM) at Korea Institute for Advanced Study. 
Open Access. This article is distributed under the terms of the Creative Commons Attribution License (CC-BY 4.0), which permits any use, distribution and reproduction in any medium, provided the original author(s) and source are credited.

\section{References}

[1] Muon G-2 collaboration, Final Report of the Muon E821 Anomalous Magnetic Moment Measurement at BNL, Phys. Rev. D 73 (2006) 072003 [hep-ex/0602035] [INSPIRE].

[2] RBC and UKQCD collaborations, Calculation of the hadronic vacuum polarization contribution to the muon anomalous magnetic moment, Phys. Rev. Lett. 121 (2018) 022003 [arXiv: 1801.07224] [INSPIRE].

[3] A. Keshavarzi, D. Nomura and T. Teubner, Muon $g-2$ and $\alpha\left(M_{Z}^{2}\right)$ : a new data-based analysis, Phys. Rev. D 97 (2018) 114025 [arXiv: 1802.02995] [INSPIRE].

[4] M. Davier, A. Hoecker, B. Malaescu and Z. Zhang, A new evaluation of the hadronic vacuum polarisation contributions to the muon anomalous magnetic moment and to $\alpha\left(m_{Z}^{2}\right)$, Eur. Phys. J. C 80 (2020) 241 [Erratum ibid. 80 (2020) 410] [arXiv:1908.00921] [INSPIRE].

[5] T. Aoyama et al., The anomalous magnetic moment of the muon in the Standard Model, arXiv:2006.04822 [INSPIRE].

[6] F. Campanario et al., Standard model radiative corrections in the pion form factor measurements do not explain the $a_{\mu}$ anomaly, Phys. Rev. D 100 (2019) 076004 [arXiv: 1903.10197] [INSPIRE].

[7] R.H. Parker, C. Yu, W. Zhong, B. Estey and H. Müller, Measurement of the fine-structure constant as a test of the Standard Model, Science 360 (2018) 191 [arXiv:1812.04130] [INSPIRE].

[8] D. Hanneke, S. Fogwell and G. Gabrielse, New Measurement of the Electron Magnetic Moment and the Fine Structure Constant, Phys. Rev. Lett. 100 (2008) 120801 [arXiv:0801.1134] [INSPIRE].

[9] G.F. Giudice, P. Paradisi and M. Passera, Testing new physics with the electron g-2, JHEP 11 (2012) 113 [arXiv:1208.6583] [inSPIRE].

[10] H. Davoudiasl and W.J. Marciano, Tale of two anomalies, Phys. Rev. D 98 (2018) 075011 [arXiv: 1806.10252] [INSPIRE].

[11] A. Crivellin, M. Hoferichter and P. Schmidt-Wellenburg, Combined explanations of $(g-2)_{\mu, e}$ and implications for a large muon EDM, Phys. Rev. D 98 (2018) 113002 [arXiv: 1807.11484] [INSPIRE].

[12] J. Liu, C.E.M. Wagner and X.-P. Wang, A light complex scalar for the electron and muon anomalous magnetic moments, JHEP 03 (2019) 008 [arXiv: 1810.11028] [INSPIRE].

[13] X.-F. Han, T. Li, L. Wang and Y. Zhang, Simple interpretations of lepton anomalies in the lepton-specific inert two-Higgs-doublet model, Phys. Rev. D 99 (2019) 095034 [arXiv: 1812.02449] [INSPIRE].

[14] M. Endo and W. Yin, Explaining electron and muon $g-2$ anomaly in SUSY without lepton-flavor mixings, JHEP 08 (2019) 122 [arXiv:1906.08768] [INSPIRE]. 
[15] M. Abdullah, B. Dutta, S. Ghosh and T. Li, $(g-2)_{\mu, e}$ and the ANITA anomalous events in a three-loop neutrino mass model, Phys. Rev. D 100 (2019) 115006 [arXiv:1907.08109] [INSPIRE].

[16] M. Bauer, M. Neubert, S. Renner, M. Schnubel and A. Thamm, Axionlike Particles, Lepton-Flavor Violation, and a New Explanation of $a_{\mu}$ and $a_{e}$, Phys. Rev. Lett. 124 (2020) 211803 [arXiv: 1908.00008] [INSPIRE].

[17] M. Badziak and K. Sakurai, Explanation of electron and muon $g-2$ anomalies in the MSSM, JHEP 10 (2019) 024 [arXiv: 1908.03607] [INSPIRE].

[18] G. Hiller, C. Hormigos-Feliu, D.F. Litim and T. Steudtner, Anomalous magnetic moments from asymptotic safety, Phys. Rev. D 102 (2020) 071901 [arXiv:1910.14062] [INSPIRE].

[19] C. Cornella, P. Paradisi and O. Sumensari, Hunting for ALPs with Lepton Flavor Violation, JHEP 01 (2020) 158 [arXiv:1911.06279] [INSPIRE].

[20] A.E. Cárcamo Hernández, Y. Hidalgo Velásquez, S. Kovalenko, H.N. Long, N.A. Pérez-Julve and V.V. Vien, Fermion spectrum and $g-2$ anomalies in a low scale 3-3-1 model, arXiv:2002.07347 [INSPIRE].

[21] N. Haba, Y. Shimizu and T. Yamada, Muon and electron $g-2$ and the origin of the fermion mass hierarchy, PTEP 2020 (2020) 093B05 [arXiv: 2002.10230] [INSPIRE].

[22] I. Bigaran and R.R. Volkas, Getting chirality right: single scalar leptoquark solution/s to the $(g-2)_{e, \mu}$ puzzle, Phys. Rev. D 102 (2020) 075037 [arXiv: 2002.12544] [INSPIRE].

[23] S. Jana, V.P.K. and S. Saad, Resolving electron and muon $g-2$ within the 2HDM, Phys. Rev. D 101 (2020) 115037 [arXiv: 2003.03386] [INSPIRE].

[24] L. Calibbi, M.L. López-Ibáñez, A. Melis and O. Vives, Muon and electron g- 2 and lepton masses in flavor models, JHEP 06 (2020) 087 [arXiv: 2003.06633] [INSPIRE].

[25] C.-H. Chen and T. Nomura, Electron and muon $g-2$, radiative neutrino mass, and $\ell^{\prime} \rightarrow \ell \gamma$ in $a \mathrm{U}(1)_{e-\mu}$ model, arXiv: 2003.07638 [INSPIRE].

[26] J.-L. Yang, T.-F. Feng and H.-B. Zhang, Electron and muon $(g-2)$ in the B-LSSM, J. Phys. G 47 (2020) 055004 [arXiv: 2003.09781] [InSPIRE].

[27] C. Hati, J. Kriewald, J. Orloff and A.M. Teixeira, Anomalies in ${ }^{8}$ Be nuclear transitions and $(g-2)_{e, \mu}$ : towards a minimal combined explanation, JHEP 07 (2020) 235 [arXiv:2005.00028] [INSPIRE].

[28] B. Dutta, S. Ghosh and T. Li, Explaining $(g-2)_{\mu, e}$, the KOTO anomaly and the MiniBooNE excess in an extended Higgs model with sterile neutrinos, Phys. Rev. D 102 (2020) 055017 [arXiv: 2006. 01319] [INSPIRE].

[29] F.J. Botella, F. Cornet-Gomez and M. Nebot, Electron and muon $g-2$ anomalies in general flavour conserving two Higgs doublets models, Phys. Rev. D 102 (2020) 035023 [arXiv:2006.01934] [INSPIRE].

[30] K.-F. Chen, C.-W. Chiang and K. Yagyu, An explanation for the muon and electron $g-2$ anomalies and dark matter, JHEP 09 (2020) 119 [arXiv: 2006.07929] [INSPIRE].

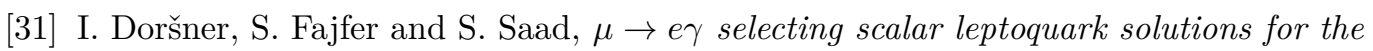
$(g-2)_{e, \mu}$ puzzles, Phys. Rev. D 102 (2020) 075007 [arXiv:2006.11624] [INSPIRE].

[32] C. Arbeláez, R. Cepedello, R.M. Fonseca and M. Hirsch, $(g-2)$ anomalies and neutrino mass, Phys. Rev. D 102 (2020) 075005 [arXiv:2007.11007] [INSPIRE]. 
[33] S. Jana, P.K. Vishnu, W. Rodejohann and S. Saad, Dark matter assisted lepton anomalous magnetic moments and neutrino masses, Phys. Rev. D 102 (2020) 075003 [arXiv: 2008.02377] [INSPIRE].

[34] H.E. Haber and G.L. Kane, The Search for Supersymmetry: Probing Physics Beyond the Standard Model, Phys. Rept. 117 (1985) 75 [INSPIRE].

[35] N. Turok and J. Zadrozny, Electroweak baryogenesis in the two doublet model, Nucl. Phys. B 358 (1991) 471 [INSPIRE].

[36] M. Trodden, Electroweak baryogenesis, Rev. Mod. Phys. 71 (1999) 1463 [hep-ph/9803479] [INSPIRE].

[37] J.E. Kim, Light Pseudoscalars, Particle Physics and Cosmology, Phys. Rept. 150 (1987) 1 [INSPIRE].

[38] J.-M. Gerard and M. Herquet, A Twisted custodial symmetry in the two-Higgs-doublet model, Phys. Rev. Lett. 98 (2007) 251802 [hep-ph/0703051] [INSPIRE].

[39] A. Broggio, E.J. Chun, M. Passera, K.M. Patel and S.K. Vempati, Limiting two-Higgs-doublet models, JHEP 11 (2014) 058 [arXiv: 1409.3199] [INSPIRE].

[40] J. Cao, P. Wan, L. Wu and J.M. Yang, Lepton-Specific Two-Higgs Doublet Model: Experimental Constraints and Implication on Higgs Phenomenology, Phys. Rev. D 80 (2009) 071701 [arXiv: 0909.5148] [INSPIRE].

[41] L. Wang and X.-F. Han, A light pseudoscalar of 2HDM confronted with muon g-2 and experimental constraints, JHEP 05 (2015) 039 [arXiv:1412.4874] [INSPIRE].

[42] V. Ilisie, New Barr-Zee contributions to $(g-2)_{\mu}$ in two-Higgs-doublet models, JHEP 04 (2015) 077 [arXiv: 1502.04199] [INSPIRE].

[43] T. Abe, R. Sato and K. Yagyu, Lepton-specific two Higgs doublet model as a solution of muon g- 2 anomaly, JHEP 07 (2015) 064 [arXiv: 1504.07059] [INSPIRE].

[44] T. Han, S.K. Kang and J. Sayre, Muon $g-2$ in the aligned two Higgs doublet model, JHEP 02 (2016) 097 [arXiv: 1511.05162] [INSPIRE].

[45] E.J. Chun and J. Kim, Leptonic Precision Test of Leptophilic Two-Higgs-Doublet Model, JHEP 07 (2016) 110 [arXiv:1605.06298] [INSPIRE].

[46] A. Cherchiglia, P. Kneschke, D. Stöckinger and H. Stöckinger-Kim, The muon magnetic moment in the 2HDM: complete two-loop result, JHEP 01 (2017) 007 [arXiv:1607.06292] [INSPIRE].

[47] A. Cherchiglia, D. Stöckinger and H. Stöckinger-Kim, Muon g- 2 in the 2HDM: maximum results and detailed phenomenology, Phys. Rev. D 98 (2018) 035001 [arXiv:1711.11567] [INSPIRE].

[48] L. Wang, J.M. Yang, M. Zhang and Y. Zhang, Revisiting lepton-specific 2HDM in light of muon g- 2 anomaly, Phys. Lett. B 788 (2019) 519 [arXiv:1809.05857] [INSPIRE].

[49] J.L. Hewett and T.G. Rizzo, Low-Energy Phenomenology of Superstring Inspired $E_{6}$ Models, Phys. Rept. 183 (1989) 193 [INSPIRE].

[50] S.D. Thomas and J.D. Wells, Phenomenology of Massive Vectorlike Doublet Leptons, Phys. Rev. Lett. 81 (1998) 34 [hep-ph/9804359] [INSPIRE]. 
[51] K. Kannike, M. Raidal, D.M. Straub and A. Strumia, Anthropic solution to the magnetic muon anomaly: the charged see-saw, JHEP 02 (2012) 106 [Erratum ibid. 10 (2012) 136] [arXiv:1111.2551] [INSPIRE].

[52] R. Dermisek and A. Raval, Explanation of the Muon g-2 Anomaly with Vectorlike Leptons and its Implications for Higgs Decays, Phys. Rev. D 88 (2013) 013017 [arXiv:1305.3522] [INSPIRE].

[53] ATLAS collaboration, A search for the dimuon decay of the Standard Model Higgs boson with the ATLAS detector, arXiv:2007.07830 [INSPIRE].

[54] CMS collaboration, Measurement of Higgs boson decay to a pair of muons in proton-proton collisions at $\sqrt{s}=13 \mathrm{TeV}$, Tech. Rep. CMS-PAS-HIG-19-006 (2020) [INSPIRE].

[55] B. Barman, D. Borah, L. Mukherjee and S. Nandi, Correlating the anomalous results in $b \rightarrow s$ decays with inert Higgs doublet dark matter and muon $(g-2)$, Phys. Rev. D 100 (2019) 115010 [arXiv:1808.06639] [INSPIRE].

[56] M. Frank and I. Saha, Muon Anomalous Magnetic Moment in Two Higgs Doublet Models with Vector-Like Leptons, arXiv:2008.11909 [INSPIRE].

[57] G.C. Branco, P.M. Ferreira, L. Lavoura, M.N. Rebelo, M. Sher and J.P. Silva, Theory and phenomenology of two-Higgs-doublet models, Phys. Rept. 516 (2012) 1 [arXiv:1106.0034] [INSPIRE].

[58] W. Grimus and L. Lavoura, The Seesaw mechanism at arbitrary order: Disentangling the small scale from the large scale, JHEP 11 (2000) 042 [hep-ph/0008179] [INSPIRE].

[59] M. Lindner, M. Platscher and F.S. Queiroz, A Call for New Physics: The Muon Anomalous Magnetic Moment and Lepton Flavor Violation, Phys. Rept. 731 (2018) 1 [arXiv: 1610.06587] [INSPIRE].

[60] J. Haller, A. Hoecker, R. Kogler, K. Mönig, T. Peiffer and J. Stelzer, Update of the global electroweak fit and constraints on two-Higgs-doublet models, Eur. Phys. J. C 78 (2018) 675 [arXiv: 1803.01853] [INSPIRE].

[61] J. Song and Y.W. Yoon, $W \gamma$ decay of the elusive charged Higgs boson in the two-Higgs-doublet model with vectorlike fermions, Phys. Rev. D 100 (2019) 055006 [arXiv: 1904.06521] [INSPIRE].

[62] ALEPH, DELPHI, L3, OPAL and SLD collaborations, LEP Electroweak Working Group, SLD Electroweak Group, SLD Heavy Flavour Group, Precision electroweak measurements on the $Z$ resonance, Phys. Rept. 427 (2006) 257 [hep-ex/0509008] [INSPIRE].

[63] A. Crivellin, F. Kirk, C.A. Manzari and M. Montull, Global Electroweak Fit and Vector-Like Leptons in Light of the Cabibbo Angle Anomaly, arXiv:2008.01113 [INSPIRE].

[64] M.E. Peskin and T. Takeuchi, A New constraint on a strongly interacting Higgs sector, Phys. Rev. Lett. 65 (1990) 964 [INSPIRE].

[65] M.E. Peskin and T. Takeuchi, Estimation of oblique electroweak corrections, Phys. Rev. D 46 (1992) 381 [INSPIRE].

[66] L. Lavoura and J.P. Silva, The Oblique corrections from vector-like singlet and doublet quarks, Phys. Rev. D 47 (1993) 2046 [inSPIRE].

[67] CMS collaboration, Search for vector-like leptons in multilepton final states in proton-proton collisions at $\sqrt{s}=13 \mathrm{TeV}$, Phys. Rev. D 100 (2019) 052003 [arXiv:1905.10853] [InSPIRE]. 
[68] E.J. Chun, Z. Kang, M. Takeuchi and Y.-L.S. Tsai, LHC $\tau$-rich tests of lepton-specific 2HDM for $(g-2)_{\mu}$, JHEP 11 (2015) 099 [arXiv: 1507.08067] [INSPIRE].

[69] E.J. Chun, S. Dwivedi, T. Mondal and B. Mukhopadhyaya, Reconstructing a light pseudoscalar in the Type-X Two Higgs Doublet Model, Phys. Lett. B 774 (2017) 20 [arXiv: 1707.07928] [INSPIRE].

[70] E.J. Chun, S. Dwivedi, T. Mondal, B. Mukhopadhyaya and S.K. Rai, Reconstructing heavy Higgs boson masses in a type $X$ two-Higgs-doublet model with a light pseudoscalar particle, Phys. Rev. D 98 (2018) 075008 [arXiv:1807.05379] [InSPIRE].

[71] ATLAS collaboration, Reconstruction and identification of boosted di- $\tau$ systems in a search for Higgs boson pairs using $13 \mathrm{TeV}$ proton-proton collision data in ATLAS, arXiv:2007.14811 [INSPIRE].

[72] E.J. Chun and T. Mondal, Searching for a Light Higgs Boson via the Yukawa Process at Lepton Colliders, Phys. Lett. B 802 (2020) 135190 [arXiv:1909.09515] [InSPIRE]. 